\title{
Discusión: La mirada sociocultural en las prácticas narrativas de niños hispanohablantes
}

\author{
Discussion: The sociocultural gaze in narrative practices of \\ Spanish-speaking children \\ Alejandra Auza Benavides ${ }^{1}$
Hospital General "Dr. Manuel Gea González", México
}

Este volumen ha reunido una serie de investigaciones elaboradas en torno al tema de la narracióninfantil.La originalidad del volumen que nos ocupa es por un lado, que se trata de trabajos en poblaciones de niños hispanohablantes que viven en diferentes culturas. Aunque en la última década han aumentado los estudios sobre la adquisición del español todavía existen muchas facetas qué estudiar para comprender mejor los procesos del desarrollo del lenguaje. Por otro lado, el volumen examina la narración mediante prácticas narrativas en América Latina. Este abordaje permite comprender la necesidad de considerar a la cultura como parte explicativa de la construcción del lenguaje.

Cada uno de los artículos que forman el volumen aporta una singular contribución para comprender el lenguaje a través de la herencia cultural, el contexto socioeconómico, el nivel educativo, la afectividad, los géneros y prácticas narrativas en sus distintas facetas tanto en niños con y sin dificultades del lenguaje. Además, se enfatiza la importancia de la información que se obtiene del contexto y cómo el niño aprende y considera la perspectiva propia y la ajena para elaborar una narración. Por tal motivo, remarcaré las aportaciones más valiosas de los trabajos y expondré la relación y las coincidencias más sobresalientes que mantienen entre sí.
La influencia de la cultura en las narraciones

Contar historias es propio de cualquier cultura del mundo. Pero la forma en que se cuenta una historia está filtrada por la cultura del individuo. La cultura tiene mucho que decir en el contenido de las historias cuando se ponen en marcha las interacciones familiares. Sparks, Carmiol y Ríos mostraron que de una manera natural, las interacciones entre las madres y sus hijos suscitan conversaciones sobre eventos pasados, convirtiéndose en una herramienta clave para que el niño construya y practique un repertorio de narraciones, dentro del que se encuentra la estructura clásica narrativa. Y es que esta estructura que tiene un desarrollo, alcanza su culmen y logra su resolución, coincide con la estructura de una conversación sobre hechos del pasado. Sparks, Carmiol y Ríos ponen en relieve que el contenido de las narraciones con estructura de punto álgido entre pequeños hablantes costarricenses influye positivamente en la producción de estructuras más complejas en forma y contenido. Esto se debe a que conversaciones de este tipo se enriquecen por las emociones y sentimientos que el niño puede manifestar a muy corta edad. Haber obtenido una estructura narrativa compleja en niños de edad preescolar sugiere que el recuerdo de estos hechos es emocionalmente significativo para los

\footnotetext{
${ }^{1}$ Alejandra Auza Benavides, Hospital General “Dr. Manuel Gea González”, México. E-mail: aauza@prodigy.net.mx Dirección postal: Av. Calzada de Tlalpan \# 4800 Col. Sección XVI, Deleg. Tlalpan, Distrito Federal CP. 14080, México.
} 
niños. Más aún, se pone en evidencia que justo en el seno de las familias y antes del comienzo escolar se germina la narración.

Así como en este estudio, la cultura de casa y el nivel socioeducativo tienen un impacto en el desarrollo narrativo de los niños, en el de Schröder, Keller y Kleis, la cultura y el género de los padres en las narraciones infantiles tienen una influencia innegable. La mirada sociocultural del trabajo lo vuelve muy novedoso, pues logra observarse que el contenido de las interacciones y la manera en que las familias de origen costarricense, mexicano y alemán les hablan a los niños generan cambios notorios. Un hallazgo llamativo es que las interacciones más elaboradas son entre madres e hijos (los padres son más escuetos al hablar con los niños) y están sujetas a un efecto bidireccional con el niño. Esto quiere decir que las madres modifican las interacciones con sus hijos a partir de cómo ellos las involucran en la conversación. Este hallazgo da cuenta de la importancia que tiene el niño como participante activo en la interacción, lo que refleja que se favorece el estilo participativo de los pequeños hablantes. Una línea que podría derivarse de este estudio sería investigar la narración entre pares de hermanos de las tres culturas y comparar si el efecto ya observado en la interacción entre padres e hijos se mantiene igual. Esta sugerencia nace a partir de otros trabajos etnográficos donde la participación activa de niños pequeños es evidente. Por ejemplo, se ha visto en familias bilingües indígenas (tzotzil-español) del sureste de México donde los miembros de una familia de cuatro generaciones y quienes han adquirido el español como primera lengua, se ajustan lingüísticamente a la elección del tzotzil del niño más pequeño de cuatro años quien también es bilingüe. A pesar de la preponderancia de hablantes de español como primera lengua en el grupo de pares de hermanos y primos, es decir, entre los miembros de su misma generación, la sintonía se establece en la familia modificando las interacciones, a favor de la integración del más pequeño (de León, 2013). Es interesante observar que el niño puede conducir una conversación, tal como se observa en las interacciones de las díadas mexicanas y las germanas mostradas por Schröder, Keller y Kleis. Esto demuestra que las familias consideran la sintonía en las conversaciones para incluir a los participantes más pequeños, pero que los patrones de interacción varían, con fuerte dependencia de la cultura subyacente.

\section{Reminiscencias, emociones y afectividad en las narraciones}

Nolivos y Leyva coinciden con el estudio de Sparks, Carmiol y Ríos, pues utilizaron la narración de reminiscencias de eventos negativos y positivos en niños chilenos que viven en condiciones socioeconómicas desfavorecidas. Estudiar a niños que no cuentan con los recursos para acceder con facilidad a la educación y a la cultura hace que la investigación adquiera un valor excepcional, pues se aportan puntos de referencia sobre el lenguaje, para una amplia porción de individuos que comparten una situación similar en América Latina. La principal contribución de Nolivos y Leyva es haber encontrado que los padres se preocupan por etiquetar las emociones y hablar de los antecedentes que provocan los estados emocionales, más que en discutir las consecuencias de los eventos negativos (comportamientos inadecuados) narrados por sus hijos. Las conversaciones sobre emociones negativas tienden a ser más extensas porque se regulan los conflictos, se evalúa el grado de intensidad del evento y se comprenden las emociones generadas. Esto tiene una implicación importante en los estudios adquisicionistas porque son estrategias que permiten generar 
más lenguaje en niños pequeños. Pero que los padres hablen poco de las consecuencias de los comportamientos inadecuados de los niños podría ser resultado de creencias arraigadas entre los adultos latinos quienes posiblemente piensen que los niños no comprenden estados mentales propios y/o ajenos. O podría especularse que se trata de actitudes del adulto frente a la construcción de la identidad psicológica del niño en la que solo las emociones que ellos creen pertinentes para sus hijos son las que son motivo de discusión. El trabajo invita a averiguar cuáles son las actitudes de los adultos en distintas comunidades con respecto a los temas que se consideran aptos para niños. Tanto el contenido como las creencias podrían ser tópicos que enriquezcan las hipótesis sobre cómo se construyen las prácticas narrativas en niños hispanohablantes.

En la misma línea de las actitudes parentales, Shiro estudió la función expresiva de la afectividad y las emociones en el discurso de hablantes bilingües, con especial énfasis en la actitud que la madre expresa al niño. El uso de la afectividad y la experiencia emocional a través del lenguaje y los cambios que elabora el hablante ha cobrado importancia en la última década. Un aspecto que llama la atención es que los hablantes adultos experimentan cambios en la expresión de sus sentimientos, en la empatía y la conciencia social que establecen con los demás (Ożańska-Ponikwia, 2012). El estado psicológico y la actitud que un individuo tiene frente a una lengua diferente a la materna pueden influir tanto en el proceso de adquisición como en la disposición para utilizarla. Esto se aprende en el seno de la familia, donde la cultura y las emociones se filtran a través del lenguaje de la madre. Al centrarse en la referencia explícita de las emociones para mostrar placer, miedo e ira, para expresar la voluntad, el deseo o la actitud epistémica expresada mediante verbos mentales, Shiro encontró que la cultura de las madres influye en las expresiones de afectividad. Por ejemplo, cuando se dirigen a sus hijos, las madres latinas expresan su afecto de acuerdo con las pautas de su propia cultura y son menos proclives que las madres angloparlantes a hacer referencia a la voluntad o al deseo. Son datos que muestran cómo el afecto y sus manifestaciones están supeditados a la cultura mostrando que tienen un peso crucial en la interacción lingüística de las madres con sus hijos desde una temprana edad.

La expresión de las emociones propias del niño también se filtra cuando se evalúan las emociones de los personajes de una historia, tal como encontraron Romero y Gómez en niños mexicanos entre 3 y 12 años de edad. El tópico cobra importancia a la luz de los estudios sobre la teoría de la mente, en la que la interpretación es una de las funciones lingüísticas y cognitivas primordiales. Al interpretar una narración se traslucen las emociones del propio narrador frente a una historia, su actitud frente a esta $y$, a la vez, se combina con el conocimiento del mundo. Para que el niño pueda evaluar, no solo se requiere de la interacción con el contenido de la historia y sus personajes, sino emplear sus recursos narrativos, gramaticales y léxicos que le faciliten la expresión de sus interpretaciones. Los resultados de estas autoras mostraron congruencia con los trabajos hechos en otras lenguas, pues no es sino hasta los siete años cuando los niños incrementan la cantidad y calidad en la evaluación narrativa. Esto sucede como consecuencia de un desarrollo más elaborado sobre la comprensión de las emociones propias y ajenas, al mismo tiempo que se afinan los recursos lingüísticos. Será digno de estudio que en un futuro, las investigadoras comparen los datos con niños de otros estratos socioeconómicos, en los que con seguridad 
estarán presentes las expresiones sobre las emociones de los personajes, pero probablemente con variaciones gramaticales y léxicas.

Habilidades macro y micro estructurales en la narración: desde el preescolar hasta la adolescencia en niños con y sin Trastorno del Lenguaje

Siguiendo con la importancia del nivel socioeconómico, Melzi, Schick y Bostwick describieron el desarrollo de las narraciones en niños hispanohablantes de bajo nivel socioeconómico que viven en Estados Unidos. El estudio de este grupo de niños es fundamental para conocer las características macro y microestructurales de las narraciones de una población creciente en ese país. Basándose en cuentos de estructura tradicional, las autoras analizaron la autonomía conversacional, la gramática de la narración y el lenguaje literario en niños de edad preescolar. El estudio de estas autoras parece coincidir en un aspecto interesante con los estudios tanto de Sparks, Carmiol y Ríos como con el de Romero y Gómez. Y es que los niños de edad preescolar se ven motivados a contar una historia cuando involucra sentimientos, pensamientos y deseos, ya sea los propios o los de los personajes, como sucede en este estudio. Como era de esperarse, cuando los niños son mayores, también lo es su complejidad estructural y gramatical. Valioso es el hecho de que compararon no solo grupos de diferentes edades sino la narración hecha por el mismo niño en diversos períodos cronológicos. La trayectoria de la narración proyecta un crecimiento esperado, pero no por menos intrigante, pues entre los tres años y medio y los cinco años y medio, el niño muestra sus habilidades lingüísticas que van desde marcas pragmáticas básicas, mayor número de referentes y frases nominales hasta conectores gramaticales en oraciones coordinadas y subordinadas.
Los niños se vuelven mejores narradores, habilidad que radica en estructurar una línea narrativa y mantener una cohesión entre las diversas acciones, los planes y la resolución que entrelazan los eventos, reflejo del desarrollo del pensamiento lógico, causal y deductivo, y consecuencia de una evolución cognitiva. Por otro lado, se va desarrollando una teoría de la mente más elaborada en la que poco a poco toman en cuenta la perspectiva del otro para incluirlo en la narración mediante información contextual y mantener la coherencia narrativa. El tema de la teoría de la mente también se refleja en el uso creciente de verbos mentales, con los que el niño comparte información sobre sus creencias y pensamientos que corren en paralelo con la narración de la historia. Los distintos aspectos de esta investigación son una clara muestra de cómo interactúan los dominios del lenguaje con la cognición en una actividad exclusivamente humana que es la de narrar historias. En ellas, el léxico y el pensamiento revelan su confabulación para volverse cada vez más complejos y continuar su ascenso en etapas tardías.

El desarrollo léxico en edades tardías adquiere un lugar preponderante en la adquisición del lenguaje dado que el crecimiento aumenta de forma vertiginosa a los seis años, edad en la que la comprensión del léxico oscila entre 6000 y 10000 palabras para convertirse entre seis y siete veces más grande cuando se inicia la etapa universitaria. Pero esta diversidad léxica puede variar significativamente, dependiendo del entorno social en el que se desenvuelve el individuo. Más aún, las habilidades de elección léxica son distintas cuando se trata de narrar oralmente o de forma escrita, dadas las restricciones particulares de las tareas en cuestión. El artículo de Hess da cuenta de estas diferencias y se enfoca en el lenguaje académico, cuya incidencia en el pensamiento abstracto $-\mathrm{y}$ 
viceversa- es fundamental. En este se condensa información cognitiva y semántica más densa que la que se emplea en otros contextos y géneros lingüísticos. Por otro lado, esta información se estructura en construcciones gramaticales más elaboradas y de mayor complejidad que hacen que este tipo de lenguaje sea atractivo como objeto de estudio. Cuando los adolescentes se enfrentan a la elección léxica que deben realizar al optar por un marco de narración oral o escrita es claro, a juzgar por el estudio de Hess, que la lengua escrita requiere que el individuo utilice una mayor densidad nominal. Las nominalizaciones son un tipo de léxico abstracto que condensa y empaqueta material conceptual idóneo para expresar estados mentales, intenciones y procesos psicológicos. Es posible que el propio uso de vocabulario abstracto y denso sea el abono de otros terrenos como las expresiones no literales, donde la abstracción y el desdoblamiento de otros significados, son el fruto fértil de géneros muy elaborados, como resulta ser la modalidad escrita de una narración. Será interesante que en el futuro se establezcan correlaciones entre el uso de la densidad léxica de los adolescentes y la cantidad de expresiones no literales utilizadas en la narración.

Hasta este punto hemos podido constatar a través de las diversas investigaciones presentadas en este volumen que si bien la narración varía entre culturas y prácticas por la forma en que miramos al mundo, también posee una estructura que parece ser fruto de una organización cognoscitiva de la mente humana. Los eventos que cualquier niño vive forman estas estructuras que son el marco conceptual para construir historias personales, anécdotas, cuentos y otro tipo de relatos (Hess \& Auza, 2013). La cultura que se aprende dentro de los contextos familiares y escolares establece las pautas para el desarrollo de las habilidades y prácticas narrativas. En algunos casos, estas habilidades están disminuidas como sucede con los niños con Trastorno Específico/Primario del Lenguaje estudiados por Coloma, Maggiolo y Pavez. El estudio reveló que la comprensión narrativa de este grupo de niños presenta problemas, comparándolos con niños de su misma edad cronológica, en preguntas tanto literales como inferenciales sobre el relato de una historia. Resulta interesante reflexionar sobre cómo la comprensión global, literal e inferencial se ven afectadas, cuando los niños deben interpretar preguntas explícitas e implícitas de un cuento. Sobre todo a la luz de diferentes aspectos de la narración que pudiesen estar implicados en la problemática de la comprensión, ya que es conocido que este grupo de niños reflejan dificultades para procesar información concerniente a nociones temporales, de causa y de motivación, lo que produce dificultades tanto en la expresión como en la comprensión de las narraciones (Colozzo, Gillam, Wood, Schnell \& Johnston, 2011). Hemos señalado a través de las voces de otros artículos en este volumen sobre la importancia de las prácticas narrativas. Este tema cobra una relevancia fundamental entre niños con dificultades del lenguaje, pues las prácticas que pudiesen parecer naturales y fácilmente asequibles por los niños con un desarrollo típico del lenguaje resultan complejas en la adquisición atípica. En muchos países latinoamericanos donde narrar hechos, historias y anécdotas es una actividad común en casa y en la escuela, sería necesario pues, apuntar hacia el ajuste de las prácticas educativas y comunicativas dirigidas a estos niños. Debería promoverse la comprensión de diversos componentes de una historia, a través de la contextualización de aspectos sutiles del lenguaje, como lo es la inferencia. Si las preguntas más difíciles son las que aluden a la culminación de la historia 
quiere decir que la interpretación de un evento completo no es exitosa para estos niños. Si bien comprenden con más facilidad la presentación y el episodio de una historia, ligados a elementos más tangibles y concretos, la resolución en cambio resulta difícil en la medida en que la idea requiere de una interpretación más abstracta. Planear herramientas terapéuticas a través de la comprensión de componentes claves de la narración será sin duda una línea para ampliar en una próxima investigación.

Gracias a las investigaciones que conforman este volumen hemos comprendido que las narraciones infantiles de hechos reales o ficticios son un sistema que involucra varias esferas del desarrollo y la cultura. La cognición, la evolución psicológica y emocional, el procesamiento de la información, la percepción sobre la perspectiva de uno mismo y del otro, la elaboración y sofisticación lingüística a través de los géneros narrativos son componentes ineludibles en los nuevos caminos de la investigación sobre narraciones de culturas latinas. Hemos podido constatar que existe una gama inagotable de factores que provocan diversidad de estilos y formas de narrar el mundo. Narrar es un tema vasto y complejo. El comienzo de futuras líneas de investigación ya está trazado.

\section{Referencias}

Colozzo, P., Gillam, R.B., Wood, M., Schnell, R.D. \& Johnston, J. (2011). Content and Form in the Narratives of Children With Specific Language Impairment. Journal of Speech, Language and Hearing Research, 54, 1609-1627.

de León, L. (2013). Interacciones bilingües en niños del México indígena: el grupo de pares como nicho de socialización lingüística. Conferencia presentada en el VII Congreso Internacional de Adquisición del Lenguaje, septiembre.

Hess, K. \& Auza, A. (2013). Las narraciones como una ventana para mirar el lenguaje y la cognición de los niños. En A. Auza \& K. Hess (Eds.) ¿Qué me cuentas? Narraciones y desarrollo lingüístico en niños bispanohablantes, (pp. 7-26) México-Querétaro: DeLaurel.

Ożańska-Ponikwia, K. (2012). What has personality and emotional intelligence to do with 'feeling different' while using a foreign language? International Journal of Bilingual Education \& Bilingualism, 15(2), 217-234. 